\title{
On the Graphs of Minimum Degree At Least 3 Having Minimum Sum-Connectivity Index
}

\author{
Wael W. Mohammed, ${ }^{1,2}$ Shahzad Ahmed, ${ }^{3}$ Zahid Raza, ${ }^{4}$ Jia-Bao Liu $\left(\mathbb{D},{ }^{5}\right.$ Farooq Ahmad, ${ }^{1,6}$ \\ and Elsayed M. Elsayed (iD ${ }^{7}$ \\ ${ }^{1}$ Department of Mathematics, Faculty of Science, University of Ha'il, Ha'il, Saudi Arabia \\ ${ }^{2}$ Department of Mathematics, Faculty of Science, Mansoura University, Mansoura 35516, Egypt \\ ${ }^{3}$ Knowledge Unit of Science, University of Management and Technology, Sialkot, Pakistan \\ ${ }^{4}$ Department of Mathematics, College of Sciences, University of Sharjah, Sharjah, UAE \\ ${ }^{5}$ School of Mathematics and Physics, Anhui Jianzhu University, Hefei 230601, China \\ ${ }^{6}$ School of Mechanical and Aerospace Engineering, NANYANG Technological University, Singapore \\ ${ }^{7}$ Department of Mathematics, Faculty of Science, King Abdulaziz University, Jeddah 21589, Saudi Arabia
}

Correspondence should be addressed to Jia-Bao Liu; liujiabaoad@163.com

Received 2 August 2021; Accepted 22 December 2021; Published 4 February 2022

Academic Editor: Tomas Vertik

Copyright (c) 2022 Wael W. Mohammed et al. This is an open access article distributed under the Creative Commons Attribution License, which permits unrestricted use, distribution, and reproduction in any medium, provided the original work is properly cited.

For a graph $G$, its sum-connectivity index is denoted by $\chi(G)$ and is defined as the sum of the numbers $(d(u)+d(v))^{-1 / 2}$ over all edges $u v$ of $G$, where $d(w)$ denotes the degree of a vertex $w \in V(G)$. In this study, we find a sharp lower bound on the sumconnectivity index of graphs having minimum degree of at least 3 under certain constraints and characterize the corresponding extremal graphs.

\section{Introduction}

In chemical graph theory, graph invariants are usually known as topological indices. Such indices play an important role in the study of quantitative structure property relationship (QSPR) and quantitative structure activity relationship (QSAR). Information about several good studied topological indices can be found in [1-7] and related references listed therein.

The well-known Randić-connectivity index (also known as the product-connectivity index), proposed by Randić [8] in 1975, is the most used topological index in QSPR and QSAR, which is defined as [8]

$$
R(G)=\sum_{u v \in E(G)}(d(u) d(v))^{-1 / 2} .
$$

The sum-connectivity index, a variant of the productconnectivity index, of a graph $G$ is defined as [9]

$$
\chi(G)=\sum_{u v \in E(G)}(d(u)+d(v))^{-1 / 2} .
$$

In the previous studies [10-12], the sum-connectivity index of several graphs of molecules was calculated. In [13], relation between the sum-connectivity index and average distance was established. Extremal results concerning minimum sum-connectivity index and matching number were obtained in [14] for trees and connected unicyclic graphs, in [15] for connected bicyclic graphs, and in [16] for cacti. Results on trees with given matching number and maximum sum-connectivity index were found in [17]. Some mathematical aspects of $\chi$ index were studied in [9], particularly for the family of trees, which contains molecular 
trees representing acyclic hydrocarbons. More detail about the $\chi$ index can be found in the recent survey [18] and recent articles $[19,20]$. In the present study, we obtained the minimum general sum-connectivity index from the family of all $n$-vertex (where $n \geq 23$ ) graphs having minimum degree of at least 3 under certain constraints.

\section{Main Results}

Theorem 1. For $n \geq 23$, let $G$ be an $n$-vertex graph with minimum degree of at least 3 , such that $G$ satisfies the following two conditions:

(i) If $G$ has a pair of adjacent vertices of degree 3, then this pair of adjacent vertices has at most one common neighbor

(ii) If $G$ contains no pair of adjacent vertices of degree 3, then $G$ has a vertex of degree 3 whose all neighbors form a triangle

Then, it holds that

$$
\chi(G) \geq \frac{3(n-3)}{\sqrt{n+2}}+\frac{3}{\sqrt{2(n-1)}},
$$

with the equality sign if and only if $G \cong K_{3}+\bar{K}_{n-3}$.

\section{Some Preliminaries Lemmas}

In this section, some lemmas are given, which play a vital role in proving the main result.

Lemma 1 (See [21]). If $e \in E(G)$ is of maximal weight, then $\chi(G)>\chi(G-e)$.

Lemma 2. If $x, y, z, w \geq 3$, then the function $f$ defined by

$$
\begin{aligned}
f(x, y, z, w)= & \frac{1}{\sqrt{x+3}}+\frac{1}{\sqrt{y+3}}+\frac{1}{\sqrt{z+3}}+\frac{1}{\sqrt{w+3}} \\
& -\frac{1}{\sqrt{x+4}}-\frac{1}{\sqrt{y+4}}-\frac{1}{\sqrt{z+4}}-\frac{1}{\sqrt{w+4}},
\end{aligned}
$$

is strictly decreasing in all $x, y, z$, and $w$ on the interval $[3, \infty)$.

Proof.

$$
\frac{\partial f}{\partial x}=\frac{-1}{2 \sqrt{(x+3)^{3}}}+\frac{1}{2 \sqrt{(x+4)^{3}}}<0,
$$

shows that the function $f$ is strictly decreasing in $x$. Because of the symmetry, we also conclude that $f$ is strictly decreasing in $y, z$, and $w$.

Lemma 3. If $n$ is an integer greater than 16 , then the function $f$ defined by $f(n)=\frac{3 n-8}{\sqrt{n+1}}-\frac{3(n-3)}{\sqrt{n+2}}+\frac{3}{\sqrt{2(n-2)}}-\frac{3}{\sqrt{2(n-1)}}+\frac{1}{\sqrt{6}}$,

is positive valued.

Proof. Clearly,

$$
\frac{3 n-5}{\sqrt{n+1}}-\frac{3 n-5}{\sqrt{n+2}}>0
$$

Also,

$$
\frac{3}{\sqrt{2 n-4}}-\frac{3}{\sqrt{2 n-2}}>0
$$

So,

$$
\frac{-3}{\sqrt{n+1}}+\frac{1}{\sqrt{6}}>0
$$

for all $n>53$.

From the above inequalities, we get that $f(n)>0$ for all $n>53$.

If $17 \leq n \leq 53$, then $f(n)>0$ by using Mathematica. Hence, $f(n)>0$ for all $n \geq 17$.

This completes the proof of the lemma.

Lemma 4. If $n$ is an integer greater than 22, then the function $f$ defined by

$$
\begin{aligned}
f(x)= & \frac{1}{\sqrt{x+3}}-\frac{2}{\sqrt{x+4}}+\frac{x-1}{\sqrt{x+n-4}} \\
& -\frac{x-1}{\sqrt{x+n-3}}, \text { where } x \geq 3,
\end{aligned}
$$

is strictly increasing on the interval $[3, \infty)$.

Proof.

$$
\begin{aligned}
\frac{\mathrm{d} f}{\mathrm{~d} x}= & \frac{-1}{2 \sqrt{(x+3)^{3}}}+\frac{1}{\sqrt{(x+4)^{3}}}+\frac{x+2 n-7}{2 \sqrt{(x+n-4)^{3}}} \\
& -\frac{x+2 n-5}{2 \sqrt{(x+n-3)^{3}}} .
\end{aligned}
$$

For $x \geq 3$, it holds that

$$
\left(\frac{7}{6}\right)^{3 / 2}<(\sqrt{2})^{2}
$$

which together with inequality $1+(1 / x+3) \leq 7 / 6$ imply that

$$
\left(1+\frac{1}{x+3}\right)^{3 / 2} \leq\left(\frac{7}{6}\right)^{3 / 2}<2
$$

which further implies that 


$$
\begin{gathered}
\frac{2}{(x+4)^{3 / 2}}-\frac{1}{(x+3)^{3 / 2}}>0 \\
\text { or } \frac{1}{(x+4)^{3 / 2}}-\frac{1}{2(x+3)^{3 / 2}}>0 .
\end{gathered}
$$

Let

$$
g(n)=\frac{x+2 n-7}{2 \sqrt{(x+n-4)^{3}}}
$$

then,

$$
\frac{\mathrm{d} g}{\mathrm{~d} n}=\frac{-2 n+x+5}{4 \sqrt{(x+n-4)^{5}}}<0,
$$

this shows that the function $g$ is decreasing in $n$.

So,

$$
\frac{x+2 n-7}{2 \sqrt{(x+n-4)^{3}}}-\frac{x+2 n-5}{2 \sqrt{(x+n-3)^{3}}}>0 .
$$

Using these inequalities in the above equation, we get

$$
\frac{\mathrm{d} f}{\mathrm{~d} x}>0 .
$$

Hence, the function $f$ is strictly increasing in $x$.

Lemma 5. Let

$$
\begin{aligned}
f(n)= & \frac{3(n-4)}{\sqrt{n+1}}-\frac{3(n-3)}{\sqrt{n+2}}+\frac{3}{\sqrt{2(n-2)}}-\frac{3}{\sqrt{2(n-1)}}+\frac{2}{\sqrt{n-1}} \\
& -\frac{2}{\sqrt{n}}+\frac{3}{\sqrt{6}}-\frac{2}{\sqrt{7}} .
\end{aligned}
$$

If $n$ is an integer greater than 13 , then $f(n)>0$.

Proof. Given,

$$
\begin{aligned}
f(n)= & \frac{3(n-4)}{\sqrt{n+1}}-\frac{3(n-3)}{\sqrt{n+2}}+\frac{3}{\sqrt{2(n-2)}}-\frac{3}{\sqrt{2(n-1)}}+\frac{2}{\sqrt{n-1}} \\
& -\frac{2}{\sqrt{n}}+\frac{3}{\sqrt{6}}-\frac{2}{\sqrt{7}} \\
> & \frac{3(n-4)}{\sqrt{n+1}}-\frac{3(n-3)}{\sqrt{n+2}}+\frac{3}{\sqrt{2(n-2)}}-\frac{3}{\sqrt{2(n-1)}} \\
& +\frac{2}{\sqrt{n-1}}-\frac{2}{\sqrt{n}}+\frac{1}{\sqrt{6}} .
\end{aligned}
$$

As

$$
\begin{gathered}
\frac{3(n-3)}{\sqrt{n+1}}-\frac{3(n-3)}{\sqrt{n+2}}>0, \\
\frac{3}{\sqrt{2(n-2)}}-\frac{3}{\sqrt{2(n-1)}}>0,
\end{gathered}
$$

also

$$
\frac{2}{\sqrt{n-1}}-\frac{2}{\sqrt{n}}>0
$$

the expression is

$$
\frac{-3}{\sqrt{n+1}}+\frac{1}{\sqrt{6}}>0
$$

for all $n>53$.

From the above inequalities, we get that $f(n)>0$ for all $n>53$.

If $14 \leq n \leq 53$, then $f(n)>0$ by using Mathematica. Hence, $f(n)>0$ for all $n \geq 14$.

Lemma 6. If $n$ is an integer greater than 22, then the function $f$ defined by

$$
\begin{aligned}
f(x)= & \frac{1}{\sqrt{x+3}}-\frac{2}{\sqrt{x+4}}+\frac{x-1}{\sqrt{x+n-3}} \\
& -\frac{x-1}{\sqrt{x+n-2}}, \text { where } x \geq 3,
\end{aligned}
$$

is strictly increasing on the interval $[3, \infty)$.

$$
\begin{aligned}
& \text { Proof. } \\
& \qquad \begin{aligned}
\frac{\mathrm{d} f}{\mathrm{~d} x}= & \frac{-1}{2 \sqrt{(x+3)^{3}}}+\frac{1}{\sqrt{(x+4)^{3}}}+\frac{x+2 n-5}{2 \sqrt{(x+n-3)^{3}}} \\
& -\frac{x+2 n-3}{2 \sqrt{(x+n-2)^{3}}} .
\end{aligned}
\end{aligned}
$$

For $x \geq 3$, it holds that

$$
\left(\frac{7}{6}\right)^{3 / 2}<(\sqrt{2})^{2}
$$

which together with inequality $1+(1 / x+3) \leq 7 / 6$ imply that

$$
\left(1+\frac{1}{x+3}\right)^{3 / 2} \leq\left(\frac{7}{6}\right)^{3 / 2}<2,
$$

which further implies that

$$
\begin{gathered}
\frac{2}{(x+4)^{3 / 2}}-\frac{1}{(x+3)^{3 / 2}}>0 \\
\text { or } \frac{1}{(x+4)^{3 / 2}}-\frac{1}{2(x+3)^{3 / 2}}>0 .
\end{gathered}
$$

Let 


$$
g(n)=\frac{x+2 n-5}{2 \sqrt{(x+n-3)^{3}}} .
$$

Then,

$$
\frac{\mathrm{d} g}{\mathrm{~d} n}=\frac{x-2 n+3}{4 \sqrt{(x+n-3)^{5}}}<0
$$

So,

$$
\frac{x+2 n-5}{2 \sqrt{(x+n-3)^{3}}}-\frac{x+2 n-3}{2 \sqrt{(x+n-2)^{3}}}>0 .
$$

Using these inequalities in the above equation, we get

$$
\frac{\mathrm{d} f}{\mathrm{~d} x}>0 .
$$

Hence, the function $f$ is strictly increasing in $x$.

Lemma 7. The function $f$ defined is by

$$
\begin{aligned}
f(n)= & \frac{3 n-14}{\sqrt{n+1}}-\frac{3(n-3)}{\sqrt{n+2}}+\frac{3}{\sqrt{2(n-2)}}-\frac{3}{\sqrt{2(n-1)}} \\
& +\frac{2}{\sqrt{n}}+\frac{3}{\sqrt{6}}-\frac{2}{\sqrt{7}} .
\end{aligned}
$$

If $n$ is an integer greater than 13, then $f(n)$ is positive valued.

Proof. Given,

$$
\begin{aligned}
f(n)= & \frac{3 n-14}{\sqrt{n+1}}-\frac{3(n-3)}{\sqrt{n+2}}+\frac{3}{\sqrt{2(n-2)}}-\frac{3}{\sqrt{2(n-1)}} \\
& +\frac{2}{\sqrt{n}}+\frac{3}{\sqrt{6}}-\frac{2}{\sqrt{7}} \\
> & \frac{3 n-14}{\sqrt{n+1}}-\frac{3(n-3)}{\sqrt{n+2}}+\frac{3}{\sqrt{2(n-2)}}-\frac{3}{\sqrt{2(n-1)}} \\
& +\frac{2}{\sqrt{n}}+\frac{1}{\sqrt{6}} .
\end{aligned}
$$

As

$$
\begin{gathered}
\frac{3(n-3)}{\sqrt{n+1}}-\frac{3(n-3)}{\sqrt{n+2}}>0, \\
\frac{3}{\sqrt{2(n-2)}}-\frac{3}{\sqrt{2(n-1)}}>0,
\end{gathered}
$$

also

$$
\frac{2}{\sqrt{n}}-\frac{2}{\sqrt{n+1}}>0
$$

$$
\frac{-3}{\sqrt{n+1}}+\frac{1}{\sqrt{6}}>0
$$

for all $n>53$.

From the above inequalities, we get that $f(n)>0$ for all $n>53$.

If $14 \leq n \leq 53$, then $f(n)>0$ by using Mathematica. Hence, $f(n)>0$ for all $n \geq 14$.

Lemma 8. If $x \geq 3, y \geq 4$, then the function $f$ defined by

$f(x, y)=\frac{1}{\sqrt{x+y}}-\frac{1}{\sqrt{x+y-1}}+\frac{y-1}{\sqrt{y+3}}-\frac{y-2}{\sqrt{y+2}}$,

is strictly increasing in $x$ and strictly decreasing in $y$.

Proof.

$$
\frac{\partial f}{\partial x}=\frac{-1}{2 \sqrt{(x+y)^{3}}}+\frac{1}{2 \sqrt{(x+y-1)^{3}}}>0 .
$$

Now,

$$
\begin{aligned}
\frac{\partial f}{\partial y}= & \frac{y+7}{2 \sqrt{(y+3)^{3}}}-\frac{y+6}{2 \sqrt{(y+2)^{3}}}+\frac{1}{\sqrt{(x+y-1)^{3}}} \\
& -\frac{1}{2 \sqrt{(x+y)^{3}}},
\end{aligned}
$$

$$
\frac{\partial^{2} f}{\partial x \partial y}=\frac{-3}{4 \sqrt{(x+y-1)^{5}}}+\frac{3}{4 \sqrt{(x+y)^{5}}}<0 .
$$

So, we have

$$
\frac{\partial f}{\partial y} \leq \frac{\partial f(3, y)}{\partial y}=\frac{y+6}{2 \sqrt{(y+3)^{3}}}-\frac{y+5}{2 \sqrt{(y+2)^{3}}} .
$$

Let

$$
g(y)=\frac{y+6}{2{\sqrt{(y+3)^{3}}}^{3}}
$$

Then,

$$
\frac{\mathrm{d} g}{\mathrm{~d} y}=\frac{-y-12}{4 \sqrt{(y+3)^{5}}}<0,
$$

which shows that $g$ is strictly decreasing in $y$.

So,

$$
\frac{y+6}{2 \sqrt{(y+3)^{3}}}-\frac{y+5}{2 \sqrt{(y+2)^{3}}}<0 .
$$

By using this inequality in the above expression, we get

SO, 


$$
\frac{\partial f}{\partial y}<0
$$

Hence, the function $f$ is strictly decreasing in $y$.

Lemma 9. Let

$f(n)=\frac{2(n-5)}{\sqrt{n+1}}-\frac{2(n-4)}{\sqrt{n+2}}+\frac{3}{\sqrt{2(n-2)}}-\frac{3}{\sqrt{2(n-1)}}+\frac{1}{\sqrt{6}}$.

If $n$ is an integer greater than 9 , then $f(n)>0$.

Proof. As

$$
\begin{aligned}
& \frac{2(n-4)}{\sqrt{n+1}}-\frac{2(n-4)}{\sqrt{n+2}}>0, \\
& \frac{3}{\sqrt{2(n-2)}}-\frac{3}{\sqrt{2(n-1)}}>0,
\end{aligned}
$$

so,

$$
\frac{-2}{\sqrt{n+1}}+\frac{1}{\sqrt{6}}>0
$$

for all $n>23$.

From the above inequalities, we get that $f(n)>0$ for all $n>23$.

If $10 \leq n \leq 23$, then $f(n)>0$ by using Mathematica.

Hence, $f(n)>0$ for all $n \geq 10$.

Lemma 10. If $x \geq 4$, then the function $f$ defined by

$$
f(x)=\frac{x}{\sqrt{x+3}}-\frac{x-1}{\sqrt{x+2}}
$$

is strictly decreasing on the interval $[4, \infty)$.

Proof.

$$
\frac{\mathrm{d} f}{\mathrm{~d} x}=\frac{x+6}{2 \sqrt{(x+3)^{3}}}-\frac{x+5}{2 \sqrt{(x+2)^{3}}} .
$$

Let

$$
g(x)=\frac{x+6}{2 \sqrt{(x+3)^{3}}} .
$$

Then,

$$
g^{\prime}(x)=\frac{-x-12}{4 \sqrt{(x+3)^{5}}}<0 .
$$

So,

$$
\frac{x+6}{2 \sqrt{(x+3)^{3}}}-\frac{x+5}{2 \sqrt{(x+2)^{3}}}<0 .
$$

Using this in the above equation, we get

$$
\frac{\mathrm{d} f}{\mathrm{~d} x}<0 .
$$

Hence, the function $f$ is strictly decreasing in $x$.

Lemma 11. If $n$ is an integer greater than 9, then the function $f$ defined by

$$
\begin{aligned}
f(n)= & \frac{4 n-14}{\sqrt{n+1}}-\frac{3(n-3)}{\sqrt{n+2}}+\frac{3}{\sqrt{2(n-2)}}-\frac{3}{\sqrt{2(n-1)}} \\
& -\frac{n-3}{\sqrt{n}}+\frac{1}{\sqrt{6}},
\end{aligned}
$$

is positive valued.

Proof. As

$$
\begin{array}{r}
\frac{3(n-3)}{\sqrt{n+1}}-\frac{3(n-3)}{\sqrt{n+2}}>0, \\
\frac{3}{\sqrt{2(n-2)}}-\frac{3}{\sqrt{2(n-1)}}>0,
\end{array}
$$

the expression is

$$
\begin{aligned}
& \frac{n-2}{\sqrt{n+1}}-\frac{n-3}{\sqrt{n}} \\
= & \sqrt{n+1}-\sqrt{n}+\frac{3}{\sqrt{n}}-\frac{3}{\sqrt{n+1}}>0 .
\end{aligned}
$$

So,

$$
\frac{-3}{\sqrt{n+1}}+\frac{1}{\sqrt{6}}>0
$$

for all $n>53$.

From the above inequalities, we get that $f(n)>0$ for all $n>53$.

If $10 \leq n \leq 53$, then $f(n)>0$ by using Mathematica.

Hence, $f(n)>0$ for all $n \geq 10$.

Lemma 12. If $x, y, z \geq 4$, then the function $f$ defined by

$$
\begin{aligned}
f(x, y, z)= & \frac{x-2}{\sqrt{x+3}}-\frac{x-3}{\sqrt{x+2}}+\frac{1}{\sqrt{x+y}}-\frac{1}{\sqrt{x+y-2}} \\
& +\frac{y-2}{\sqrt{y+3}}-\frac{y-3}{\sqrt{y+2}}+\frac{1}{\sqrt{y+z}}-\frac{1}{\sqrt{y+z-2}} \\
& +\frac{z-2}{\sqrt{z+3}}-\frac{z-3}{\sqrt{z+2}}+\frac{1}{\sqrt{x+z}}-\frac{1}{\sqrt{x+z-2}},
\end{aligned}
$$


is decreasing in all $x, y$ and $z$.

Proof.

$$
\begin{aligned}
\frac{\partial f}{\partial x}= & \frac{x+8}{2 \sqrt{(x+3)^{3}}}-\frac{x+7}{2 \sqrt{(x+2)^{3}}}-\frac{1}{2 \sqrt{(x+y)^{3}}}-\frac{1}{2 \sqrt{(x+z)^{3}}} \\
& +\frac{1}{2 \sqrt{(x+y-2)^{3}}}+\frac{1}{2 \sqrt{(x+z-2)^{3}}},
\end{aligned}
$$

which implies

$$
\begin{gathered}
\frac{\partial^{2} f}{\partial x \partial y}=\frac{3}{4 \sqrt{(x+y)^{5}}}-\frac{3}{4 \sqrt{(x+y-2)^{5}}}<0, \\
\frac{\partial^{2} f}{\partial x \partial z}=\frac{3}{4 \sqrt{(x+z)^{5}}}-\frac{3}{4 \sqrt{(x+z-2)^{5}}}<0 .
\end{gathered}
$$

Since $\partial^{2} f / \partial x \partial y<0$ and $\partial^{2} f / \partial x \partial z<0$, it holds that

$$
\begin{aligned}
\frac{\partial f}{\partial x} & \leq \frac{\partial f(x, 4,4)}{\partial x} \\
& =\frac{x+8}{2 \sqrt{(x+3)^{5}}}-\frac{x+5}{2 \sqrt{(x+2)^{5}}}-\frac{1}{\sqrt{(x+4)^{3}}} .
\end{aligned}
$$

Let

$$
F(x)=\frac{x+8}{2 \sqrt{(x+3)^{3}}}-\frac{x+5}{2 \sqrt{(x+2)^{3}}}-\frac{1}{\sqrt{(x+4)^{3}}},
$$

where $x \geq 4$. We note that

$$
F(x)=h(x+1)-h(x)+g(x)-g(x+1),
$$

where

$$
\begin{aligned}
& h(x)=\frac{x+5}{2 \sqrt{(x+2)^{3}}}, \\
& g(x)=\frac{1}{\sqrt{(x+3)^{3}}} .
\end{aligned}
$$

The functions $h$ and $g$ are continuous as well as differentiable on the closed interval $\left[x_{o}, x_{o}+1\right]$ for every fixed number $x_{o} \geq 4$.

By virtue of Cauchy's mean value theorem, we observe that for every $x_{o}$, there exists a number $c_{x o} \in\left(x_{o}, x_{o}+1\right)$, such that

$$
\begin{aligned}
& \frac{h\left(x_{o}+1\right)-h\left(x_{o}\right)}{g\left(x_{o}+1\right)-g\left(x_{o}\right)}=\frac{h^{\prime}\left(c_{x o}\right)}{g^{\prime}\left(c_{x o}\right)} \\
= & \left(\frac{c_{x o}+3}{c_{x o}+2}\right)^{5 / 2}\left(\frac{c_{x o}+11}{6}\right),
\end{aligned}
$$

which is greater than one, and hence,

$$
F\left(x_{o}\right)=h\left(x_{o}+1\right)-h\left(x_{o}\right)+g\left(x_{o}\right)-g\left(x_{o}+1\right)<0,
$$

because the function $g$ is decreasing.

Therefore, for all $x \geq 4$, the function $F(x)$ is negative valued. Using $F(x)<0$ in the above equation, we get

$$
\frac{\partial f}{\partial x}<0
$$

This shows that the function $f$ is decreasing in $x$. By symmetry, it follows that

$$
\begin{aligned}
& \frac{\partial f}{\partial y}<0, \\
& \frac{\partial f}{\partial z}<0,
\end{aligned}
$$

which means that the function $f$ is decreasing in $y$ and $z$. Hence, the function $f$ is decreasing in $x, y$, and $z$.

\section{Proof of Theorem 1}

Proof. We will prove the result by induction on $n$. For $n=23$, by using the AutoGraphiX system [22], we find that $K_{3}+\bar{K}_{n-3}$ has the maximum $\chi$ value among all $n$-vertex graphs having a minimum degree of at least 3 , which implies that the result is true for $n=23$. Now, we suppose that the theorem holds for all those $k$-vertex graphs which satisfy all the constraints of the theorem, where $23 \leq k \leq n-1$.

If minimum degree of $G$ is at least 4 , then we take $v_{1} v_{2} \in E(G)$, such that $d\left(v_{1}\right)+d\left(v_{2}\right) \leq d(u)+d(v)$ for all $u v \in E(G)$. Clearly, the graph $G-v_{1} v_{2}$ has minimum degree of at least 3, and from Lemma 1, it follows that $\chi(G)>\chi\left(G-v_{1} v_{2}\right)$. Hence, it is sufficient to assume that the minimum degree of $G$ is 3 . Next, we consider all possible cases.

Case 1:

$G$ contains at least one pair of adjacent vertices having degree 3 without common neighbors

Let $u_{1}, u_{2} \in V(G)$ be two adjacent vertices having degree 3 without any common neighbor. Let $v_{1}$ and $v_{2}$ be the neighbors of $u_{1}$ different from $u_{2}$, and $w_{1}, w_{2}$ be the 
neighbors of $u_{2}$ different from $u_{1}$. In this case, the vertex degrees $d\left(v_{1}\right), d\left(v_{2}\right), d\left(w_{1}\right)$, and $d\left(w_{2}\right)$ satisfy the inequality $3 \leq d\left(v_{1}\right), d\left(v_{2}\right), d\left(w_{1}\right), d\left(w_{2}\right) \leq n-2$. By setting $G_{1} \cong G-\left\{u_{1}\right\}+\left\{u_{2} v_{1}, u_{2} v_{2}\right\}$ and then by using Lemma 2 , the inductive hypothesis, and Lemma 3 , we have

$$
\begin{aligned}
\chi(G)= & \chi\left(G_{1}\right)+\frac{1}{\sqrt{d\left(u_{1}\right)+d\left(u_{2}\right)}}+\frac{1}{\sqrt{d\left(u_{1}\right)+d\left(v_{1}\right)}}+\frac{1}{\sqrt{d\left(u_{1}\right)+d\left(v_{2}\right)}} \\
& +\frac{1}{\sqrt{d\left(u_{2}\right)+d\left(w_{1}\right)}}-\frac{1}{\sqrt{d\left(u_{2}\right)+1+d\left(w_{1}\right)}}+\frac{1}{\sqrt{d\left(u_{2}\right)+d\left(w_{2}\right)}} \\
& -\frac{1}{\sqrt{d\left(u_{2}\right)+1+d\left(w_{2}\right)}}-\frac{1}{\sqrt{d\left(u_{2}\right)+1+d\left(v_{1}\right)}}-\frac{1}{\sqrt{d\left(u_{2}\right)+1+d\left(v_{2}\right)}} \\
= & \chi\left(G_{1}\right)+\frac{1}{\sqrt{6}}+\frac{1}{\sqrt{3+d\left(v_{1}\right)}}+\frac{1}{\sqrt{3+d\left(v_{2}\right)}} \\
& +\frac{1}{\sqrt{3+d\left(w_{1}\right)}}-\frac{1}{\sqrt{4+d\left(w_{1}\right)}}+\frac{1}{\sqrt{3+d\left(w_{2}\right)}} \\
& -\frac{1}{\sqrt{4+d\left(w_{2}\right)}}-\frac{1}{\sqrt{4+d\left(v_{1}\right)}}-\frac{1}{\sqrt{4+d\left(v_{2}\right)}} \\
\geq & \frac{3(n-4)}{\sqrt{n+1}+\frac{3}{\sqrt{2(n-2)}}+\frac{4}{\sqrt{n+1}}}-\frac{4}{\sqrt{n+2}}+\frac{1}{\sqrt{6}} \\
> & \frac{3(n-3)}{\sqrt{n+2}+\frac{3}{\sqrt{2(n-1)}} .}
\end{aligned}
$$

Case 2:

$G$ contains at least one pair of adjacent vertices of degree 3 with a common neighbor

Let $u_{1}, u_{2} \in V(G)$ be two adjacent vertices of degree 3 having a common neighbor $v$. Let $v_{1}$ be the neighbor of $u_{1}$ different from $u_{2}$, and $v$ and $w_{1}$ be the neighbors of $u_{2}$ different from $u_{1}, v$.
Subcase i:

Let $d(v)=3$, and there is an edge between $v_{1}$ and $v$. Clearly, it holds that $3 \leq d\left(v_{1}\right) \leq n-2$ and $3 \leq d\left(w_{1}\right) \leq n-3$. If we take $G_{2} \cong G-\left\{u_{1}\right\}+\left\{u_{2} v_{1}\right.$, $\left.w_{1} v\right\}$, then by using Lemma 4 , the inductive hypothesis, and Lemma 5, we get

$$
\begin{aligned}
\chi(G)= & \chi\left(G_{2}\right)+\frac{1}{\sqrt{d\left(u_{1}\right)+d\left(u_{2}\right)}}+\frac{1}{\sqrt{d\left(u_{1}\right)+d(v)}}+\frac{1}{\sqrt{d\left(u_{1}\right)+d\left(v_{1}\right)}} \\
& -\frac{1}{\sqrt{d\left(u_{2}\right)+d\left(v_{1}\right)}}-\frac{1}{\sqrt{d(v)+d\left(w_{1}\right)+1}} \\
& +\frac{1}{\sqrt{d\left(u_{2}\right)+d\left(w_{1}\right)}}-\frac{1}{\sqrt{d\left(u_{2}\right)+d\left(w_{1}\right)+1}}
\end{aligned}
$$




$$
\begin{aligned}
& +\sum_{z \in N\left(w_{1}\right) \backslash\left\{u_{2}\right\}}\left(\frac{1}{\sqrt{d(z)+d\left(w_{1}\right)}}-\frac{1}{\sqrt{d(z)+d\left(w_{1}\right)+1}}\right) \\
\geq & \chi\left(G_{2}\right)+\frac{2}{\sqrt{6}}-\frac{1}{\sqrt{3+d\left(w_{1}\right)+1}}+\frac{1}{\sqrt{3+d\left(w_{1}\right)}} \\
& -\frac{1}{\sqrt{3+d\left(w_{1}\right)+1}}+\frac{d\left(w_{1}\right)-1}{\sqrt{n-4+d\left(w_{1}\right)}}-\frac{d\left(w_{1}\right)-1}{\sqrt{n-4+d\left(w_{1}\right)+1}} \\
\geq & \chi\left(G_{2}\right)+\frac{2}{\sqrt{n-1}}-\frac{2}{\sqrt{n}}+\frac{3}{\sqrt{6}}-\frac{2}{\sqrt{7}} \\
\geq & \frac{3(n-4)}{\sqrt{n+1}}+\frac{3}{\sqrt{2(n-2)}}+\frac{2}{\sqrt{n-1}}-\frac{2}{\sqrt{n}}+\frac{3}{\sqrt{6}}-\frac{2}{\sqrt{7}} \\
> & \frac{3(n-3)}{\sqrt{n+2}}+\frac{3}{\sqrt{2(n-1)}} .
\end{aligned}
$$

Subcase ii:

Let $d(v)=3$, and there is an no edge between $v_{1}$ and $v$.

Clearly, it holds that $3 \leq d\left(v_{1}\right) \leq n-3$. If $G_{3} \cong G-$ $\left\{u_{1}\right\}+\left\{u_{2} v_{1}, v_{1} v\right\}$, then by using Lemma 6 , the induction hypothesis, and Lemma 7, we obtained

$$
\begin{aligned}
& \chi(G)=\chi\left(G_{3}\right)+\frac{1}{\sqrt{d\left(u_{1}\right)+d\left(u_{2}\right)}}+\frac{1}{\sqrt{d\left(u_{1}\right)+d(v)}}+\frac{1}{\sqrt{d\left(u_{1}\right)+d\left(v_{1}\right)}} \\
& -\frac{1}{\sqrt{d\left(u_{2}\right)+d\left(v_{1}\right)+1}}-\frac{1}{\sqrt{d(v)+d\left(v_{1}\right)+1}} \\
& +\sum_{z \in N\left(v_{1}\right) \backslash\left\{u_{1}\right\}}\left(\frac{1}{\sqrt{d(z)+d\left(v_{1}\right)}}-\frac{1}{\sqrt{d(z)+d\left(v_{1}\right)+1}}\right) \\
& \geq \chi\left(G_{3}\right)+\frac{2}{\sqrt{6}}+\frac{1}{\sqrt{3+d\left(v_{1}\right)}}-\frac{1}{\sqrt{3+d\left(v_{1}\right)+1}} \\
& -\frac{1}{\sqrt{3+d\left(v_{1}\right)+1}}+\frac{d\left(v_{1}\right)-1}{\sqrt{n-3+d\left(v_{1}\right)}}-\frac{d\left(v_{1}\right)-1}{\sqrt{n-3+d\left(v_{1}\right)+1}} \\
& \geq \chi\left(G_{3}\right)+\frac{2}{\sqrt{n}}-\frac{2}{\sqrt{n+1}}+\frac{3}{\sqrt{6}}-\frac{2}{\sqrt{7}} \\
& \geq \frac{3(n-4)}{\sqrt{n+1}}+\frac{3}{\sqrt{2(n-2)}}+\frac{2}{\sqrt{n}}-\frac{2}{\sqrt{n+1}}+\frac{3}{\sqrt{6}}-\frac{2}{\sqrt{7}} \\
& >\frac{3(n-3)}{\sqrt{n+2}}+\frac{3}{\sqrt{2(n-1)}} .
\end{aligned}
$$

Subcase iii: 
Let $d(v) \geq 4$, and there is an edge between $v_{1}$ and $v$. Clearly, it holds that $3 \leq d\left(v_{1}\right) \leq n-2$. If we take
$G_{4} \cong G-u_{1}+u_{2} v_{1}$, then by using Lemma 8 , the inductive hypothesis, and Lemma 9 , we have

$$
\begin{aligned}
\chi(G)= & \chi\left(G_{4}\right)+\frac{1}{\sqrt{d\left(u_{1}\right)+d\left(u_{2}\right)}}+\frac{1}{\sqrt{d\left(u_{1}\right)+d(v)}}+\frac{1}{\sqrt{d\left(u_{1}\right)+d\left(v_{1}\right)}}-\frac{1}{\sqrt{d\left(u_{2}\right)+d\left(v_{1}\right)}} \\
& +\frac{1}{\sqrt{d\left(u_{2}\right)+d(v)}}-\frac{1}{\sqrt{d\left(u_{2}\right)+d(v)-1}}+\frac{1}{\sqrt{d\left(v_{1}\right)+d(v)}}-\frac{1}{\sqrt{d\left(v_{1}\right)+d(v)-1}} \\
& +\sum_{z \in N(v) \backslash\left\{u_{1}, v_{1}, v_{2}\right\}}\left(\frac{1}{\sqrt{d(v)+d(z)}}-\frac{1}{\sqrt{d(v)-1+d(z)}}\right) \\
\geq & \chi\left(G_{4}\right)+\frac{1}{\sqrt{6}}+\frac{2}{\sqrt{3+d(v)}}-\frac{1}{\sqrt{3+d(v)-1}}+\frac{1}{\sqrt{d\left(v_{1}\right)+d(v)}}-\frac{1}{\sqrt{d\left(v_{1}\right)+d(v)-1}} \\
& +\frac{d(v)-3}{\sqrt{d(v)+d(z)}}+\frac{d(v)-3}{\sqrt{d(v)-1+d(z)}} \\
\geq & \chi\left(G_{4}\right)+\frac{n-1}{\sqrt{n+2}}-\frac{n-2}{\sqrt{n+1}}+\frac{1}{\sqrt{6}} \\
\geq & \frac{3(n-4)}{\sqrt{n+1}}+\frac{3}{\sqrt{2(n-2)}}+\frac{n-1}{\sqrt{n+2}}-\frac{n-2}{\sqrt{n+1}}+\frac{1}{\sqrt{6}} \\
> & \frac{3(n-3)}{\sqrt{n+2}+\frac{3}{\sqrt{2(n-1)}}} .
\end{aligned}
$$

Subcase iv:

Let $d(v) \geq 4$, and there is no edge between $v_{1}$ and $v$. $G_{5} \cong G-u_{1}+u_{2} v_{1}$, then by using Lemma 10 , the Clearly, it holds that $3 \leq d\left(v_{1}\right) \leq n-3$. If we take

$$
\begin{aligned}
\chi(G)= & \chi\left(G_{5}\right)+\frac{1}{\sqrt{d\left(u_{1}\right)+d\left(u_{2}\right)}}+\frac{1}{\sqrt{d\left(u_{1}\right)+d\left(v_{1}\right)}}+\frac{1}{\sqrt{d\left(u_{1}\right)+d(v)}}-\frac{1}{\sqrt{d\left(u_{2}\right)+d\left(v_{1}\right)}}+\frac{1}{\sqrt{d\left(u_{2}\right)+d(v)}}-\frac{1}{\sqrt{d\left(u_{2}\right)+d(v)-1}} \\
& +\sum_{z \in N(v) \backslash\left\{u_{1}, u_{2}\right\}}\left(\frac{1}{\sqrt{d(v)+d(z)}}-\frac{1}{\sqrt{d(v)-1+d(z)}}\right) \\
\geq & \chi\left(G_{5}\right)+\frac{1}{\sqrt{6}}+\frac{2}{\sqrt{3+d(v)}}-\frac{1}{\sqrt{3+d(v)-1}}+\frac{d(v)-2}{\sqrt{d(v)+d(z)}}-\frac{d(v)-2}{\sqrt{d(v)-1+d(z)}} \\
\geq & \chi\left(G_{5}\right)+\frac{n-2}{\sqrt{n+1}}-\frac{n-3}{\sqrt{n}}+\frac{1}{\sqrt{6}} \geq \frac{3(n-4)}{\sqrt{n+1}}+\frac{3}{\sqrt{2(n-2)}}+\frac{n-2}{\sqrt{n+1}}-\frac{n-3}{\sqrt{n}}+\frac{1}{\sqrt{6}} . \\
> & \frac{3(n-3)}{\sqrt{n+2}}+\frac{3}{\sqrt{2(n-1)}} .
\end{aligned}
$$

Case 3: 
$G$ does not contain any pair of adjacent vertices of degree 3

Let $u \in V(G)$ be a vertex of degree 3 having neighbors $u_{1}, u_{2}$, and $u_{3}$, such that $u_{1} u_{2}, u_{1} u_{3}, u_{2} u_{3} \in E(G)$; then clearly, $d\left(u_{1}\right), d\left(u_{2}\right), d\left(u_{3}\right) \geq 4$. If we take $G_{6} \cong G-\{u\}$, then by induction hypothesis and by using Lemma 12 , we have

$$
\begin{aligned}
& \chi(G)=\chi\left(G_{6}\right)+\frac{1}{\sqrt{d(u)+d\left(u_{1}\right)}}+\frac{1}{\sqrt{d(u)+d\left(u_{2}\right)}}+\frac{1}{\sqrt{d(u)+d\left(u_{3}\right)}} \\
& +\frac{1}{\sqrt{d\left(u_{1}\right)+d\left(u_{2}\right)}}-\frac{1}{\sqrt{d\left(u_{1}\right)+d\left(u_{2}\right)-2}}+\frac{1}{\sqrt{d\left(u_{1}\right)+d\left(u_{3}\right)}} \\
& -\frac{1}{\sqrt{d\left(u_{1}\right)+d\left(u_{3}\right)-2}}+\frac{1}{\sqrt{d\left(u_{2}\right)+d\left(u_{3}\right)}}-\frac{1}{\sqrt{d\left(u_{2}\right)+d\left(u_{3}\right)-2}} \\
& +\sum_{z \in N\left(u_{1}\right) \backslash\left\{u, u_{2}, u_{3}\right\}}\left(\frac{1}{\sqrt{d\left(u_{1}\right)+d(z)}}-\frac{1}{\sqrt{d\left(u_{1}\right)-1+d(z)}}\right) \\
& +\sum_{z \in N\left(u_{2}\right) \backslash\left\{u, u_{1}, u_{3}\right\}}\left(\frac{1}{\sqrt{d\left(u_{2}\right)+d(z)}}-\frac{1}{\sqrt{d\left(u_{2}\right)-1+d(z)}}\right) \\
& +\sum_{z \in N\left(u_{3}\right) \backslash\left\{u, u_{1}, u_{2}\right\}}\left(\frac{1}{\sqrt{d\left(u_{3}\right)+d(z)}}-\frac{1}{\sqrt{d\left(u_{3}\right)-1+d(z)}}\right) \\
& \geq \chi\left(G_{6}\right)+\frac{1}{\sqrt{3+d\left(u_{1}\right)}}+\frac{1}{\sqrt{3+d\left(u_{2}\right)}}+\frac{1}{\sqrt{3+d\left(u_{3}\right)}} \\
& +\frac{1}{\sqrt{d\left(u_{1}\right)+d\left(u_{2}\right)}}-\frac{1}{\sqrt{d\left(u_{1}\right)+d\left(u_{2}\right)-2}}+\frac{1}{\sqrt{d\left(u_{1}\right)+d\left(u_{3}\right)}} \\
& -\frac{1}{\sqrt{d\left(u_{1}\right)+d\left(u_{3}\right)-2}}+\frac{1}{\sqrt{d\left(u_{2}\right)+d\left(u_{3}\right)}}-\frac{1}{\sqrt{d\left(u_{2}\right)+d\left(u_{3}\right)-2}} \\
& +\frac{d\left(u_{1}\right)-3}{\sqrt{d\left(u_{1}\right)+3}}-\frac{d\left(u_{1}\right)-3}{\sqrt{d\left(u_{1}\right)+3-1}}+\frac{d\left(u_{2}\right)-3}{\sqrt{d\left(u_{2}\right)+3}} \\
& -\frac{d\left(u_{2}\right)-3}{\sqrt{d\left(u_{2}\right)+3-1}}+\frac{d\left(u_{3}\right)-3}{\sqrt{d\left(u_{3}\right)+3}}-\frac{d\left(u_{3}\right)-3}{\sqrt{d\left(u_{3}\right)+3-1}} \\
& \geq \chi\left(G_{6}\right)+\frac{3(n-3)}{\sqrt{n+2}}-\frac{3(n-4)}{\sqrt{n+1}}+\frac{3}{\sqrt{2(n-1)}}-\frac{3}{\sqrt{2(n-2)}} \\
& \geq \frac{3(n-4)}{\sqrt{n+1}}+\frac{3}{\sqrt{2(n-2)}}+\frac{3(n-3)}{\sqrt{n+2}}-\frac{3(n-4)}{\sqrt{n+1}}+\frac{3}{\sqrt{2(n-1)}}-\frac{3}{\sqrt{2(n-2)}} \\
& =\frac{3(n-3)}{\sqrt{n+2}}+\frac{3}{\sqrt{2(n-1)}} \text {. }
\end{aligned}
$$

We observe that the equation

$$
\chi(G)=\frac{3(n-3)}{\sqrt{n+2}}+\frac{3}{\sqrt{2(n-1)}}
$$


holds if and only if

$$
d\left(u_{1}\right)=d\left(u_{2}\right)=d\left(u_{3}\right)=n-1 .
$$

This completes the proof of Theorem 1.

\section{Concluding Remarks}

We have characterized the minimum sum-connectivity index from the family of all $n$-vertex graphs having minimum degree at least 3, under certain constraints. More precisely, we have been able to show that $K_{3}+\bar{K}_{n-3}$ is the only graph having minimal sum-connectivity index in the family of all those $n$-vertex (where $n \geq 23$ ) graphs which have a minimum degree of at least 3 and satisfy the following two conditions:

(i) If $G$ has a pair of adjacent vertices of degree 3, then this pair of adjacent vertices has at most one common neighbor

(ii) If $G$ contains no pair of adjacent vertices of degree 3, then $G$ has a vertex of degree 3 whose all neighbors form a triangle.

\section{Data Availability}

The data used to support the findings of this study are included within the article.

\section{Conflicts of Interest}

The authors declare that they have no conflicts of interest.

\section{Acknowledgments}

This research was funded by Scientific Research Deanship at University of Ha'il, Saudi Arabia, through project number RG-20 050.

\section{References}

[1] A. Ali and Z. Du, "On the difference between atom-bond connectivity index and Randić index of binary and chemical trees," International Journal of Quantum Chemistry, vol. 117, no. 23, Article ID e25446, 2017.

[2] A. Ali, S. Elumalai, and T. Mansour, "On the symmetric division deg index of molecular graphs," MATCH Communications in Mathematical and in Computer Chemistry, vol. 83, no. 1, pp. 193-208, 2020.

[3] A. Emanuel, T. Doslić, and A. Ali, "Two upper bounds on the weighted Harary indices," Discrete Mathematics Letters, vol. 1, pp. 21-25, 2019.

[4] Y. Ma, S. Cao, Y. Shi, I. Gutman, M. Dehmer, and B. Furtula, "From the connectivity index to various Randictype descriptors," MATCH Communications in Mathematical and in Computer Chemistry, vol. 80, no. 1, pp. 85-106, 2018.

[5] I. Milovanović, M. Matejić, and E. Milovanović, "A note on the general zeroth-order Randić coindex of graphs," Contributions to Mathematics, vol. 1, pp. 17-21, 2020.

[6] K. Xu, M. Liu, K. C. Das, I. Gutman, and B. Furtula, "A survey on graphs extremal with respect to distance-based topological indices," MATCH Communications in Mathematical and in Computer Chemistry, vol. 71, no. 3, pp. 461-508, 2014.

[7] L. Zhong and Q. Qian, "The minimum general sum-connectivity index of trees with given matching number," Bulletin of the Malaysian Mathematical Sciences Society, vol. 43, no. 2, pp. 1527-1544, 2020.

[8] M. Randić, "Characterization of molecular branching," Journal of the American Chemical Society, vol. 97, no. 23, pp. 6609-6615, 1975.

[9] B. Zhou and N. Trinajstić, "On a novel connectivity index," Journal of Mathematical Chemistry, vol. 46, no. 4, pp. 12521270, 2009.

[10] M. Cancan, S. Ediz, S. Ediz, and M. R. Farahani, "On vedegree atom-bond connectivity, sum-connectivity, geometric-arithmetic and harmonic indices of copper oxide," Eurasian Chemical Communications, vol. 2, no. 5, pp. 641-645, 2020.

[11] M. R. Farahani, "On the Randić and sum-connectivity index of nanotubes," Analele Universitatii de Vest, Timisoara Seria Matematica Informatica, vol. 2, pp. 39-46, 2013.

[12] W. Gao, M. R. Farahani, and M. Imran, "About the Randić connectivity, modify Randić connectivity and sum-connectivity indices of titania nanotubes $\mathrm{TiO}_{2}(\mathrm{~m}, \mathrm{n})$," Acta Chimica Slovenica, vol. 64, no. 1, pp. 256-260, 2017.

[13] V. Lokesha, B. S. Shetty, P. Raju, and P. S. Ranjini, "Relation between sum-connectivity index and average distance of trees," International journal of Mathematical Combinatorics, vol. 4, pp. 92-98, 2015.

[14] Z. Du, B. Zhou, and N. Trinajstić, "Minimum general sumconnectivity index of unicyclic graphs," Journal of Mathematical Chemistry, vol. 48, no. 3, pp. 697-703, 2010.

[15] Z. Du and B. Zhou, "On sum-connectivity index of bicyclic graphs," The Bulletin of the Malaysian Mathematical Society Series 2, vol. 35, no. 1, pp. 101-117, 2012.

[16] F. Ma and H. Deng, "On the sum-connectivity index of cacti," Mathematical and Computer Modelling, vol. 54, no. 1-2, pp. 497-507, 2011.

[17] Z. Zhu and W. Zhang, "Trees with a given order and matching number that have maximum general sum-connectivity index," ARS Combinatoria, vol. 128, pp. 439-446, 2016.

[18] A. Ali, L. Zhong, and I. Gutman, "Harmonic index and its generalizations: extremal results and bounds," $M A T C H$ Communications in Mathematical and in Computer Chemistry, vol. 81, pp. 249-311, 2019.

[19] S. Ahmed, "On the maximum general sum-connectivity index of trees with a fixed order and maximum degree," Discrete Mathematics Algorithms and Applications, vol. 13, no. 4, Article ID 2150042, 2021.

[20] A. Ali, S. Ahmed, Z. Du, W. Gao, and M. A. Malik, "On the minimal general sum-connectivity index of connected graphs without pendant vertices," IEEE Access, vol. 7, pp. 136743136751, 2019.

[21] S. Wang, Z. Bo, and N. Trinajstic, "On the sum-connectivity index," Filomat, vol. 25, no. 3, pp. 29-42, 2011.

[22] G. Caporossi and P. Hansen, "Variable neighborhood search for extremal graphs: 1 the AutoGraphiX system," Discrete Mathematics, vol. 212, no. 1-2, pp. 29-44, 2000. 